\title{
Transmit and Receive Antenna Selection Based Resource Allocation for Self-Backhaul 5G Massive MIMO HetNets
}

\author{
Farah Akif ${ }^{1}$, Aqdas Malik ${ }^{1}$, Ijaz Qureshi ${ }^{2}$, and Ayesha Abassi ${ }^{1}$ \\ ${ }^{1}$ Department of Electrical Engineering, International Islamic University, Pakistan \\ ${ }^{2}$ Department of Electrical Engineering, Air University, Pakistan
}

\begin{abstract}
With the advancement in wireless communication technology, the ease of accessibility and increasing coverage area is a major challenge for service providers. Network densification through Small cell Base Stations (SBS) integration in Heterogeneous Networks (HetNets) promises to improve network performance for cell edge users. Since providing wired backhaul for small cells is not cost effective or practical, the third-Generation Partnership Project (3GPP) has developed architecture for self-backhaul known as Integrated Access and Backhaul (IAB) for Fifth Generation (5G). This allows for Main Base Station (MBS) resources to be shared between SBS and MBS users. However, fair and efficient division of MBS resources remains a problem to be addressed. We develop a novel transmit antenna selection/partitioning technique for taking advantage of IAB 5G standard for Massive Multiple Input Multiple Output (MIMO) HetNets. Transmit antenna resources are divided among access for MBS users and for providing wireless backhaul for SBS. We develop A Genetic Algorithm (GA) based Transmit Antenna Selection (TAS) scheme and compare with random selection, eigenvalue-based selection and bandwidth portioning. Our analysis show that GA based TAS has the ability to converge to an optimum antenna subset providing better rate coverage. Furthermore, we also signify the performance of TAS based partitioning over bandwidth partitioning and also show user association can also be controlled using number of antennas reserved for access or backhaul.
\end{abstract}

Keywords: Antenna selection, Massive MIMO, heterogeneous networks, genetic algorithm.

Received January 9, 2020; accepted January 13, 2021

https://doi.org/10.34028/iajit/18/6/2

\section{Introduction}

In these past couple of decades, we have observed exponential growth in cellular network traffic. Evolution of smart handheld devices and applications demand high end data rates in wireless communication. Massive increase in number of mobile users and Internet of Things (IoT) will make existing cellular network overcrowded and under efficient. In this context, heterogeneous networks coupled with massive Multiple Input Multiple Output (MIMO) systems offer the opportunity to increase network coverage and capacity by incorporating small cells within macro base station coverage area [1].

However, small cells require reliable backhaul in order to ensure user coverage. With the increase in number of Small cell Base Stations (SBS) in heterogeneous networks, it is becoming increasingly difficult to provide wired backhaul for them. Not only it is difficult to deploy SBS with wired backhaul but also cost of cable does not justify the expected benefits.

With the emergence of $5 \mathrm{G}$ standard for Integrated Access and Backhaul (IAB) [23], wireless backhaul also known as self-backhaul, has become an emerging solution for this problem. The idea is that SBS will utilize spectrum resources of Macro Base Station (MBS) for establishing a wireless connection with the backhaul.
In a way the MBS can offload some traffic to SBS without compromising on quality and promising better Quality of Service (QoS) for cell edge.

Massive MIMO (M. MIMO) technology offers high spectral efficiency by using hundreds of antennas to serve multiple users simultaneously in time and frequency resources. Due to very selective and narrowband beamforming, massive MIMO is potentially capable of interference avoidance [3].

Antenna Selection (AS) technology for massive MIMO was introduced for reducing the hardware complexity and cost of the system. In AS, a subset of antennas is selected that is capable of providing best possible Signal to Interference and Noise Ratio (SINR) [8] with the objective of reducing the number of Radio Frequency (RF) chains and hence hardware cost.

In this paper, we develop analytical framework for wireless self-backhaul using heuristic techniques which provides important insights for development of predeployment analysis for IAB for $5 \mathrm{G}$ HetNets.

\subsection{Related Work}

Research regarding heterogeneous networks has gained pace with the realization of $5 \mathrm{G}$ technology. Resource allocation is one of the key issues in multi-tier heterogeneous networks. Gerasimenko et al. [10] study 
heterogeneous cloud radio access network for cross cell resource management as well as coordination. Since the proposed work deals with transmit antenna selectionbased resource allocation for self-backhaul networks, hence, the literature survey presented here provides latest work being done in the said areas.

In [22] a two-tier heterogeneous network is considered and a downlink resource allocation and user association algorithms are proposed keeping in view the energy efficiency of the network which is partially achieved by putting unused SBS in sleep mode.

Like work done in [10], the Han et al. [11] also consider a mobile edge cloud to act as a central controller for intra-tier cooperation in heterogeneous networks. Probability of connectivity, load balancing and energy efficiency is optimized to improve network performance.

Similar to previous work, Wang et al. [30] define a software defined controller for heterogeneous network for handover management considering aeronautical scenario.

An energy efficient resource allocation scheme is presented in [28] for Non-Orthogonal Multiple Access (NOMA) HetNets. They develop power allocation scheme using bisection method for an imperfect CSI (Channel State Information).

In [14] cognitive small cells are introduced to maximize throughput using fairness-based resource allocation scheme. They combine cognitive radio and small cell technology to improve throughput.

Chai et al. [6] jointly discuss user association, cache portioning and content placement for Device To Device (D2D) heterogeneous networks. Their objective is to improve transmission performance where the sub problems are iteratively solved.

Papazafeiropoulos et al. [21], study the impact of base station cooperation in two tier heterogeneous network with user association. They develop a Software Defined Network (SDN) for controlling various resource allocation and user association tasks.

Zhou et al. [35] develop a delay-aware strategy for improving performance of services provided by heterogeneous network, while using Multi-access Edge Computing (MEC) for deciding user association.

User scheduling and association is jointly addressed in [9]. They use alternating direction method multiplier to develop an algorithm for both user and base station side, where a user can associate with more than one base station.

Sharma et al. [27] discusses full duplex self-backhaul for heterogeneous networks where coverage expressions are derived using stochastic geometry for downlink connections.

$\mathrm{Xu}$ et al. [33] discuss the inter and intra-tier interference for self-backhaul heterogeneous network where regularized zero forcing precoding is used at downlink to avoid uplink interference.

Nguyen et al. [20] the authors separate backhaul and access using time-spectrum transmission accommodation and buffering protocol for improving small cell performance.

Generally, antenna selection is employed in massive MIMO systems to reduce the overall requirement of RF chains. In this context, the Tang and Nie [29] study antenna selection while considering capacity maximization where rectangular maximum volume sub matrices are used for optimum selection.

Single and multi cell cooperative massive MIMO antenna selection scheme is presented in [12] where antenna subsets are sequentially selected to find the ones that provide the most sum rate.

Makki et al. [18] consider finite number of transmit and receive antennas for a massive MIMO network and develop antenna selection scheme using genetic algorithm.

Taking inspiration from cognitive radio networks, $[31,33]$ perform resource allocation between main cell and small cells by allowing small cells to take advantage of bandwidth holes where no licensed user is connected in the main cell. In a way small cells opportunistically use the licensed spectrum in the absence of licensed users.

In this paper we emphasize the fact that only increase in bandwidth does not guarantee a uniform increase in capacity, but in fact, the number of antennas plays an important role in keeping the Signal to Noise Ratio (SNR) consistent with increasing bandwidth as shown 2.5. We present a novel technique by using Transmit Antenna Selection (TAS) for maintaining an SINR threshold. This technique involves dividing total number of MBS antennas into two partitions. Where one partition is reserved for the user equipment associated with MBS and the second partition is reserved for providing wireless backhaul link for the SBS. The former link is generally referred to as access link while the later one is referred as backhaul link. We use nature inspired Genetic Algorithm (GA) as tool for antenna selection. GA is an optimization and search tools [25] based on principles of natural selection and genetics. GA has been used for various aspects of antenna designing [18] including transmit antenna selection where only the selected antennas are connected pre-selected number of RF chains to reduce the overall system cost. However, the related work of GA for antenna designing $[5,7,13,15,17,24,26]$ does not take into account use of the entire spatial diversity and neither does any work take into account selection of antennas for controlling QoS like it is being done in the proposed work while [2] provides another novel application of GA for IoT (internet of things) however, the application does not target the problem under discussion.

Marinello et al. [19] present a Particle swarm Optimization (PSO) based approach towards antenna selection for energy efficiency. Cai et al. [4] adopt a neural network based approach for optimizing antenna selection problem. Zhong et al. [34] propose a deep 
learning-based antenna selection where antennas were trained for maximizing capacity. The authors present a bandwidth partitioning based scheme for antenna selection in [25]. Similar work is presented in [16] for software defined radio by selecting antenna for improving capacity.

The literature surveyed highlights an important gap where the role of TAS for resource allocation with IAB has been overlooked.

\subsection{Contribution}

\subsubsection{Model Development for IAB Enabled M. MIMO TAS}

In this paper we develop a scalable analytical framework for performance analysis of IAB enabled TAS for two tier HetNets. We consider a two-tier HetNet having MBS at the center and surrounded by SBS at the edge of MBS range boundary, hence, SBS are effectively expanding the range of MBS. Users are randomly located in two tiers in a single macro cell for developing clear understanding of the system, however the model can be extended to incorporate effect of multiple macro cells. It is assumed that MBS spatial resources, i.e., total number of transmit antennas are divided for access and backhaul link using random selection, equal selection and GA based selection. Moreover, we also show that user association can also be controlled by controlling the antenna densities (here we consider it equal to number of antennas) for either access or backhaul as it directly effects the data rate.

\subsubsection{Association and Rate Coverage Modelling}

Performance evaluation and comparisons between different strategies mentioned above are carried out using rate coverage which basically represents the maximum achievable data rate for access and backhaul and any selection mechanism. We also show how capacity and number of transmit antennas are related to each other in a massive MIMO system. Two important parameters namely user association and signal to SINR are also analysed for access and backhaul links.

\subsubsection{System Design Insights}

The related work in section 1.1 does not demonstrate use of TAS for efficiently managing access and backhaul rates or providing an architecture for enabling IAB. In this work we demonstrate the usability of TAS for IAB and provide comparison with similar techniques. Usability of proposed design is validated by providing rate coverage and mean rate analysis with respect to SINR and partitioning factor.

The results demonstrate that the proposed TAS technique out performs the existing schemes including PSO scheme as presented in [12]. Moreover, it is also conclusively shown that TAS partitioning provides better rate coverage compared to bandwidth partitioning scheme of [13] with number of MBS antennas and mobile users.

\section{System Model}

\subsection{Downlink Massive MIMO HetNet Topology}

We consider a two-tier heterogeneous cellular network, where each MBS is equipped with a massive number of antennas and associated circuitry. The users in each cell are categorized according to their location as follows:

- Macro User Equipment (MUE): represents users associated with MBS only which cannot be offloaded to SBS.

- Free User Equipment (FUE): represents users that can be associated with either MBS or SBS. These users can be offloaded to SBS for load balancing of MBS.

- Small cell User Equipment (SUE): represents users that are associated with SBS only.

Although we consider a single cell for analysis, but for the sake of clarity and reference for our future work, we consider that MBSs in each cell are distributed according to Homogeneous Poisson Point Process (HPPP) $\emptyset_{m}$ with density $\lambda_{m}$. Since, MBS is equipped with a massive number of antennas, so, here we define the density of MBS antennas in single cell for access and backhaul as $\lambda_{a}$ and $\lambda_{b}$ respectively. Where both the densities depend on the number (which is also a representation of gain, all antennas are equal gain) of antenna subset selected for access and backhaul.

The SBS in second tier are spatially distributed according to HPPP $\emptyset_{s}$ with density $\lambda s$. The transmit power of MBS is represented by $P$ and each MBS is equipped with $\mathrm{M}$ antennas and serves $N$ access and backhaul connections where

$$
\begin{gathered}
N<M \\
N=N_{a}+N_{b}
\end{gathered}
$$

The Macro User Equipment (MUE) in tier 1 and small Cell User Equipment (SUE) in tier 2 are distributed according to HPPP $\emptyset_{1}$ and $\emptyset_{2}$ respectively and with respective densities $\lambda_{1}$ and $\lambda_{2}$. Moreover, users in each tier and SBS have single antenna.

The network is open access for FUE which can choose between connecting to MBS or SBS, hence, contributing largely to load balancing of MBS. Let $M a$ $M_{a}$ represent the number of MBS antennas reserved for access communication i.e., Down Link (DL) of MUE and $M_{b}$ represent the number of MBS antennas reserved for backhaul communication. Table 1 provides a summary of major notations used in this paper. 
Table 1. Notation summary.

\begin{tabular}{|c|c|}
\hline Notation & Description \\
\hline $\mathrm{M}$ & Total number of MBS antennas \\
\hline$M_{a}, M_{b}$ & $\begin{array}{c}\text { Number of MBS antenna for access } \\
\text { and backhaul }\end{array}$ \\
\hline$\lambda_{m}, \lambda_{s}$ & MBS and SBS BS densities \\
\hline$\lambda_{a}, \lambda_{b}$ & $\begin{array}{c}\text { MBS antenna subset density for access } \\
\text { and backhaul }\end{array}$ \\
\hline$\alpha$ & pathloss \\
\hline$P_{m a}, P_{m b}$ & $\begin{array}{c}\text { MBS transmit power for access } \\
\text { and backhaul antenna subset }\end{array}$ \\
\hline$T_{a}, T_{b}$ & Association weights for access and backhaul \\
\hline$I_{i, x o}, I_{j, a}, I_{j, b}, I_{r, S}$ & $\begin{array}{c}\text { Interference neighboring MBS, access and } \\
\text { backhaul antenna and SBS }\end{array}$ \\
\hline$\beta_{a}, \beta_{b}$ & Access and backhaul link gains \\
\hline$W_{a}, W_{b}$ & Access and backhaul bandwidth \\
\hline$N_{o}$ & Noise power \\
\hline$B_{a}, B_{b}$ & Access and backhaul bias \\
\hline$C_{x, a}, C_{x, b}$ & Association region for access and backhaul \\
\hline$R_{a}, R_{b}$ & Access and backhaul rate coverage \\
\hline$S I N R_{a}, S I N R_{b}, S I N R_{s u}$ & $\begin{array}{c}\text { Access and backhaul link SINR(Signal to } \\
\text { Interference plus Noise Ratio, SBS SINR }\end{array}$ \\
\hline$N_{a}, N_{b}$ & Load on access and backhaul \\
\hline$P_{a, t o t a l}, P_{b, t o t a l}$ & Total Transmit Power of access and backhaul \\
\hline$E E_{a}, E E_{b}$ & Energy Efficiency of access and backhaul \\
\hline
\end{tabular}

\subsection{MBS and SBS User Association}

First, we find the user association probability for user in tier-2 as it will define the total load on access and backhaul communication on top of tier- 1 and tier- 2 users. We modify the user association in [15]. The FUE will be associated with either tier-1 or tier-2 if;

$$
k=\arg \max _{j \in k} P_{j} B_{j} r_{j}^{-\alpha}
$$

Where $k \in\{1,2\}$ represent first and second tier. $r_{j}$ represents minimum distance from a user to its nearest Base Station (BS) in $j^{\text {th }}$ tier, where $j$ represents either first or third tier, $a>2$ is the pathloss exponent.

So, a typical FUE will be associated with either tier-1 or tier-2 with the probability in Equations (4) and (5) derived from modification in [32]

$$
\begin{aligned}
& A_{a}=\frac{\lambda_{a} T_{a}^{2 / \alpha}}{\lambda_{a} T_{a}^{2 / \alpha}+\lambda_{b} T_{b}^{2 / \alpha}} \\
& A_{b}=\frac{\lambda_{b} T_{b}^{2 / \alpha}}{\lambda_{a} T_{a}^{2 / \alpha}+\lambda_{b} T_{b}^{2 / \alpha}}
\end{aligned}
$$

We will now update (3), (4), and (5) by incorporating association weights $T_{a}, T_{b}$ for access and backhaul respectively. These weights are adjustable so that any network requirement can be met. Consider $Z_{a}$ as the distance between a user and antenna subset $M_{a}$, hence, a user will be connected to access if;

$$
\begin{aligned}
& k_{a}=\arg \max _{M_{a} \in M} T_{a} Z_{a}^{-\alpha} \\
& k_{b}=\arg \max _{M_{b} \in M} T_{b} Z_{b}^{-\alpha}
\end{aligned}
$$

if $T_{a} \gg T_{b}$ more users will be associated with access else $T_{b} \gg T_{a}$ more users will be associated with SBS

Let us consider the following three cases.
- Case 1: $T_{a}=T_{b}=1$ then the user will be associated with the nearest of the two either $M_{a}$ or $M_{b}$.

- Case 2: $T_{a}=P_{m a} B_{a}$ and $T_{b}=P_{m b} B_{b}$ uses maximum bias power to association decision, it's a typical technique used for cell range expansion. Here $B_{a}, B_{b}$ represent bias for access and backhaul respectively.

- Case 3: $B_{a}=B_{b}=1$ shows that user association is based on maximum receiver power.

\subsection{Association Region}

- Definition 1. Association region of subset of $M$ is the region of the plane where all users are served by either $M_{a}$ or $M_{b}$ MBS antenna subset. Association regions for $M_{a}$ and $M_{b}$ located at $x_{a}$ and $x_{b}$ are given as:

$$
C_{x a}=\left\{y_{a} \in \mathbb{R}^{2}:\left\|y_{a}-x_{a}\right\| \leq\left(T_{a}\right)^{1 / \alpha}\left\|y_{a}-X_{a}^{*}\left(y_{a}\right)\right\|^{\alpha}\right.
$$

Where

$$
\begin{gathered}
X_{a}^{*}\left(y_{a}\right)=\arg \min _{x_{a} \in \emptyset_{m}}\left\|y_{a}-x_{a}\right\| \\
C_{x b}=\left\{y_{b} \in \mathbb{R}^{2}:\left\|y_{b}-x_{b}\right\| \leq\left(T_{b}\right)^{1 / \alpha}\left\|y_{b}-X_{b}^{*}\left(y_{b}\right)\right\|^{\alpha}\right.
\end{gathered}
$$

Where

$$
X_{b}^{*}\left(y_{b}\right)=\arg \min _{x_{b} \in \Phi_{m}}\left\|y_{b}-x_{b}\right\|
$$

\subsection{Access and Backhaul SINR}

We consider universal frequency reuse which means that a user will not only receive the desired signal but will also receive interfering signal from neighboring cells.

We focus our analysis on FUE where the users have the option of either getting connected to SBS and be a part of backhaul communication or get connected to MBS and be part of the access communication. The Signal to Interference plus Noise Ratio (SINR) for both access and backhaul connections and also, SINR of user connect to SBS will also be calculated.

The SINR of FUE served by MBS at $x$ is given by; Where neighboring MBS is located at $x_{o}$

$$
\operatorname{SINR} R_{a}=\frac{\frac{P_{m a} a}{N_{a}} \beta_{a} x^{-\alpha}}{\sum_{i=1}^{L} I_{i, x}+\sum_{j=1}^{M_{b}} I_{j, b}+\sum_{r=1}^{S} I_{r, S}+N_{o} W_{a}}
$$

$L$ is number of neighboring MBS and $S$ is number of SBS.

The SIR of FUE which is served by an SBS in tier-2 at location $z$ is given by;

$$
\operatorname{SINR}_{S u}=\frac{\frac{P_{S}}{N_{s} \beta_{s} z^{-\alpha}}}{\sum_{i=1}^{L} I_{i, x o}+\sum_{j=1}^{M} I_{j, m}+\sum_{r=1}^{S} I_{r, S}+N_{o}}
$$

The SIR between MBS to SBS DL in backhaul can be written as;

$$
\operatorname{SINR}_{b}=\frac{\frac{P_{m b}}{N_{b}} \beta_{b} y^{-\alpha}}{\sum_{i=1}^{L} I_{i, x o}+\sum_{j=1}^{M} I_{j, a}+\sum_{r=1}^{S} I_{r, s}+N_{o} W_{b}}
$$




\subsection{Capacity Vs Number of Transmit Antennas}

A major selling point for future networks is higher bandwidth which is translated as higher capacity. However, this increase in capacity is not linear. Consider an Additive White Gaussian Noise (AWGN) communication system operating at $W \mathrm{~Hz}$ with capacity given as:

$$
R=\frac{W}{N} \log _{2}\left(1+P \beta M / N_{o} W\right)
$$

Where $N$ is the number of users (load), $P$ (watts) is transmit antenna power, $\beta$ is the path gain and $N_{o}$ is the Power Spectral Density (PSD) of noise. Generally, $\frac{P \beta}{N_{o} W}$ is referred to as SNR. From above equation, apparently increasing $W$ should increase $C$ but this increase in bandwidth will also increase noise i.e., the product $N_{o} W$. Hence, in order to make capacity and bandwidth relation linear, the SNR must be kept constant. This is where massive MIMO systems showcase their advantage. In massive MIMO $\beta=\beta_{1} M$, where $M$ is the number of transmit antennas. Hence, careful selection of number of transmit antennas can ensure a linear increase in capacity with increase in bandwidth.

\subsection{Rate Coverage}

- Definition 2. It is the maximum achievable data rate in the downlink between access link of MBS and its users $R_{a}$ and MBS and SBS backhaul $R_{b}$ which can mathematically be expressed as:

$$
\begin{aligned}
R_{a} & =\frac{W_{a}}{N_{a}} \log _{2}\left(1+\operatorname{SINR}_{a} M_{a}\right) \\
R_{b} & =\frac{W_{b}}{N_{b}} \log _{2}\left(1+\operatorname{SINR}_{b} M_{b}\right)
\end{aligned}
$$

Where $N_{a}$ and $N_{b}$ represent the load (number of users) on access and backhaul links. Figure 1 shows the relationship between number of transmit antennas and data rate.

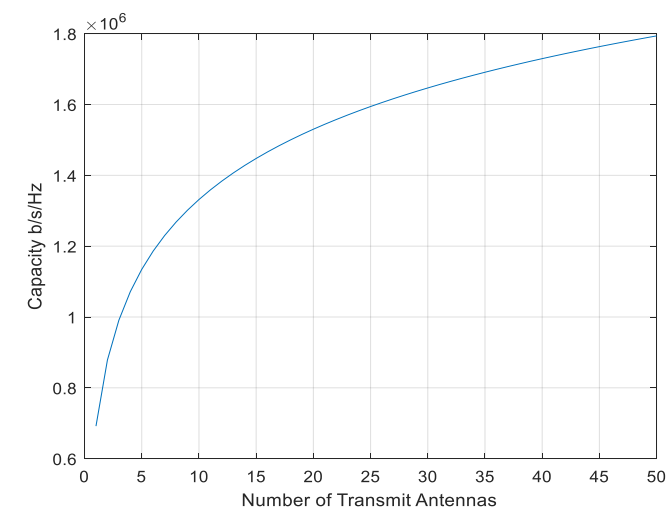

Figure 1. Effect of number of transmit antennas on data rate.

\section{Resource Allocation Based on TAS}

Here we are going to present three novel TAS selection schemes for resource allocation in Self-Backhaul HetNets (SBH).

\subsection{Offload Favouring Random Selection}

In this case, we base TAS selection on the MBS and SBS antenna density such that more users are encouraged to associate with SBS and hence offload MBS. While density of SBS will remain constant after deployment unless one malfunctions or turns off temporarily, whereas, MBS antenna density within a cell can be varied for access and backhaul links by changing the number of MBS antennas associated with each of the above links. The antennas are divided based on their channel state where higher eigenvalues of the channel matrix correspond to antennas reserved for backhaul and vice versa.

In order to encourage more backhaul links over access for MBS, we define the following condition for MBS antenna subsets;

$$
\begin{aligned}
\lambda_{b} & >\lambda_{a} \\
\text { s.t.SIR } & \geq \text { Threshold }
\end{aligned}
$$

We are considering a user in tier-2 which is in the coverage area of both SBS and MBS. We are interested in offloading MBS to provide more resources for backhaul. For this purpose, greater number of MBS antenna subset will be assigned for backhaul communication to increase the channel gain between MBS and SBS for backhaul; i.e.

$$
\begin{aligned}
& g_{z, b}>g_{x, a} \\
& M_{b}>M_{a}
\end{aligned}
$$

Conventionally, user association with either MBS or SBS is based on maximum received power which is controlled using a bias value to encourage load balancing. Here, we are interested in associating a user with the BS offering greater channel gain which can be controlled using TAS.

\subsection{Equal Selection}

This is the simplest form of selection where equal number of MBS antennas are reserved for access and backhaul. The selection of antennas for the two links is randomly done unlike the eigenvalue-based selection of the previous case. The selection in this case can be simply represented as:

$$
M_{a}=M_{b}=M / 2
$$

\subsection{GA Based Selection}

Since antenna subset selection has an exponential number of solutions with respect to number of transmit antennas, thus making it an NP problem. We consider antenna subset selection optimization using Genetic Algorithm (GA). The flow chart of GA based antenna selection is shown in Figure 2 and the steps involved in GA based selection are as follows:

1. Population: consider a total of $\mathrm{M}$ transmit antennas at MBS and $M_{b}$ the total number of antennas selected for backhaul link, so there are $M \mathbb{C}_{M_{b}}$ 
possible combinations. Let $M_{b x}$ be the number of all possible antenna subsets i.e., possible subset solutions known as chromosomes, then $\left\{\mathrm{M}_{b 1}, \ldots \ldots M_{b x}\right\}$ each represents a gene per chromosome. Population size of $\mathrm{M}$ is randomly generated and each chromosome is $\mathrm{M}$ bit long. The $\mathrm{M}$-bit chromosome string consisting of 0 's and 1's (with 1's representing selected antennas) will have a maximum of $\mathrm{M}_{\mathrm{a}} 1$ 's in case population is generated for access link antenna selection and $\mathrm{M}_{\mathrm{b}} 1$ 's in case of backhaul link. Population $=\left\{x_{1}, x_{2}, \ldots x_{M}\right\}$ where each $\mathrm{x}$ (chromosome) represents an antenna subset.

2. Fitness Function: calculate the fitness function $f(x)$ for each chromosome in the population based on randomly assigned SINR to each link.

$$
f(x)=W /{ }_{N} \log _{2}(1+\operatorname{SINR}(x))
$$

3. Selecting Parents: select fittest individuals by choosing the pair with highest fitness function to let them pass their genes to the next generation. It means selecting antenna subsets from the random population that give best fitness values.

4. Making a Crossover: from selected pair of chromosomes a new population of $\mathrm{M}$ elements is generated. Here, the selected subsets of antennas are used for generating a new population.

5. Making a Mutation: mutate some bits in chromosomes at random in new population.

6. Replace current population with new population.

7. Stopping criteria: maximum number of iterations. Go to step 2.

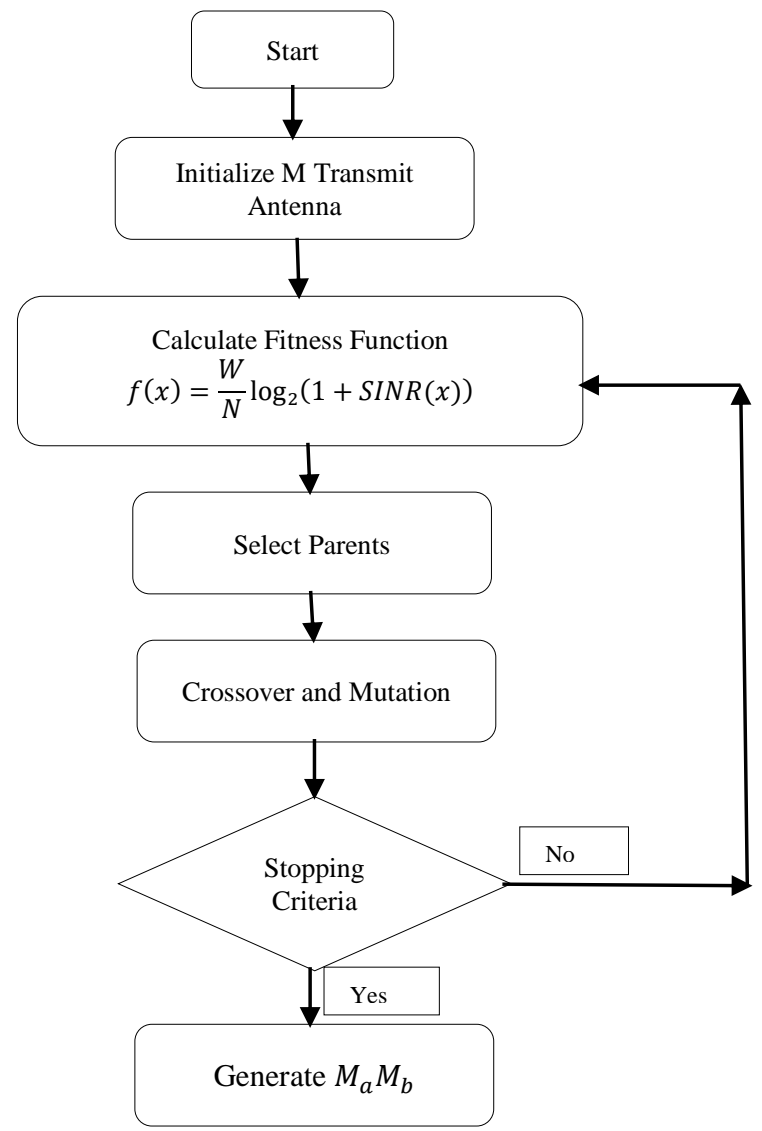

Figure 2. Flow Chart of GA based antenna selection.

\section{Performance Metrics}

\subsection{Rate Coverage Probability}

- Definition 3. It is defined as the probability that the maximum achievable data rate $R_{a}$ and $R_{b}$ of access and backhaul links, respectively, exceeds a certain threshold rate of $\rho$.

Generally, coverage probability can be represented for a user as [32] which is modified below for proposed scenario;

$$
\begin{aligned}
& p_{c a}=\mathbb{P}\left(R_{a}>\rho_{a}\right) \\
& p_{c b}=\mathbb{P}\left(R_{b}>\rho_{a}\right)
\end{aligned}
$$

Similarly, outage probability is given as:

$$
\begin{aligned}
& p_{\text {outa }}=1-p_{c a} \\
& p_{\text {outb }}=1-p_{c b}
\end{aligned}
$$

So, coverage probability of a user being served by MBS in tier-1 can be found by using (16);

$$
\begin{gathered}
p_{c a}=\mathbb{P}\left[\frac{W_{a}}{N_{a}} \log _{2}\left(1+\operatorname{SINR}_{a} M_{a}\right)>\rho_{a}\right] \\
p_{c a}=\mathbb{P}\left(\operatorname{SINR}_{a}>\frac{2^{\rho^{N} N / W_{a-1}}}{M_{a}}\right)
\end{gathered}
$$

Similarly, the coverage probability of a user being served by SBS in tier-2 can be found using (17);

$$
\begin{gathered}
p_{c b}=\mathbb{P}\left[\frac{W_{b}}{N_{b}} \log _{2}\left(1+\operatorname{SINR}_{b} M_{b}>\rho_{b}\right]\right. \\
p_{c b}=\mathbb{P}\left(\operatorname{SINR}_{b}>\frac{2^{\rho_{b} N_{b} / W_{b-1}}}{M_{b}}\right)
\end{gathered}
$$

Higher coverage probability reinforces the reliability of the access or backhaul link or in simpler terms it means a better Quality of Experience (QoE) for users while providing the promised QoS as depicted by rate coverage probability.

We now evaluate the coverage probabilities for various antenna selection schemes discussed in section 3.

The rate coverage probability for a massive MIMO HetNet with IAB for a target rate of $\rho$ is given as:

$$
\begin{aligned}
& p_{c}=p_{c a}+p_{c b} \\
& p_{c}=\mathbb{P}\left\{\left(\operatorname{SINR}_{a}+\operatorname{SINR}_{b}\right)>\left[\begin{array}{c}
\left.\left(\frac{2 N_{a} / W_{a}-1}{M_{a}}\right)+\right] \\
\left(\frac{2^{\rho N_{b} / W_{b-1}}}{M_{b}}\right)
\end{array}\right]\right\}
\end{aligned}
$$

For equal selection, $M_{a}=M_{b}=M / 2$ and assuming equal bandwidth:

$p_{c}=\mathbb{P}\left\{\left(\operatorname{SINR}_{a}+\operatorname{SINR}_{b}\right)>\frac{2}{M}\left(2^{2\left(N_{a}+N_{b}\right) \rho / W}-2\right)\right\}$

For offload favoring and GA based selection, the rate coverage probability is given as; 


$$
\begin{aligned}
& p_{c}=\mathbb{P}\left\{\left(\operatorname{SINR}_{a}+\operatorname{SINR}_{b}\right)\right. \\
& >\frac{1}{M_{a} M_{b}}\left[M_{b}\left(2^{\rho N_{a} / W_{a}}-1\right)\right. \\
& \left.\left.+M_{a}\left(2^{\rho N_{b} / W_{b}}-1\right)\right]\right\}
\end{aligned}
$$

\subsection{Energy Efficiency}

We will now derive the expressions for power model and energy efficiency model for access and backhaul links. The power model for access is given as:

$$
P_{a, \text { total }}=\left\{\frac{\frac{P_{m a}}{\delta\left(1-\sigma_{\text {feed }}\right)}+P_{\text {cir }}+P_{\text {sta }}}{\left(1-\sigma_{D C}\right)\left(1-\sigma_{M S}\right)\left(1-\sigma_{c o o l}\right)}\right\}
$$

Where $\delta$ is the power amplifier efficiency, $P_{c i r}$ is the power consumption of RF chains which consists power consumption from Digital to Analog Converter (DAC) $\left(P_{D C}\right)$, mixer $(P m i x)$, filter at transmitter $\left(P_{\text {filt }}\right)$, and frequency synthesizer (Psyn).

While lossy factors of antenna consist of losses in feeder $\left(\sigma_{\text {feed }}\right)$, DC-DC power supply $\left(\sigma_{D C}\right)$, main power supply $\left(\sigma_{M S}\right)$, and active cooling system $\left(\sigma_{\text {cool }}\right)$.

Similarly, power model for backhaul link is given as:

$$
P_{b, \text { total }}=\left\{\frac{\frac{P_{m b}}{\delta\left(1-\sigma_{\text {feed }}\right)}+P_{\text {cir }}+P_{\text {sta }}}{\left(1-\sigma_{D C}\right)\left(1-\sigma_{M S}\right)\left(1-\sigma_{c o o l}\right)}\right\}
$$

The energy efficiency model for access link is given as:

$$
E E_{a}=\frac{R_{a}}{P_{a, t o t a l}}
$$

Likewise, the energy efficiency for backhaul link is given as:

$$
E E_{b}=\frac{R_{b}}{P_{b, \text { total }}}
$$

\subsection{Mean Best Cost}

We calculated the mean capacity for Proposed Genetic Algorithm (PGA) and PSO based algorithms for 30 runs of each algorithm.

\subsection{Standard Deviation}

Standard Deviation (SD) was also calculated for the data generated from PGA and PSO algorithm which was used for describing variation in mean data values as well for calculating the Standard Error of Means (SEM).

\subsection{Standard Error of Means}

The SEM was used to measure deviation in sample's mean compared to population mean. It was used to measure the accuracy of the data sample being analysed.

\section{Simulation and Results}

\subsection{Rate Coverage Trends}

To establish a benchmark and to gauge the accuracy of analysis, we analyse the network capacity for access and backhaul links with and without Channel State Information (CSIT) in Figures 3 and 4. Water pouring algorithm is used for capacity analysis with CSIT. An improvement in capacity is observed with increase in number of transmit antennas. System parameters used for simulation are presented in Table 2.

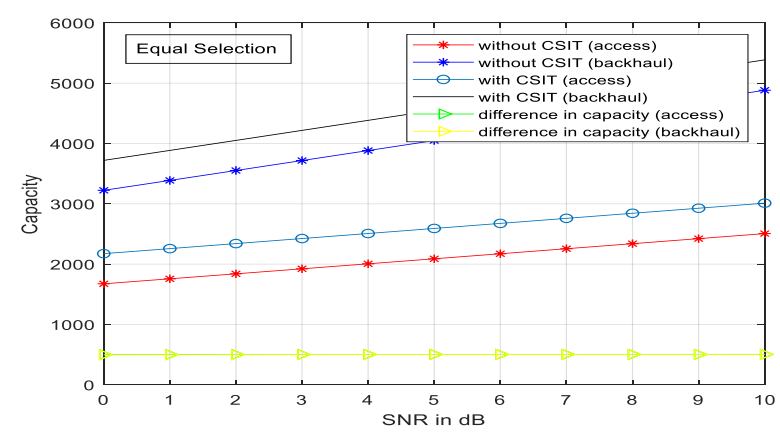

Figure 3. Effect on capacity with equal antenna selection for access and backhaul, with and without CSIT.

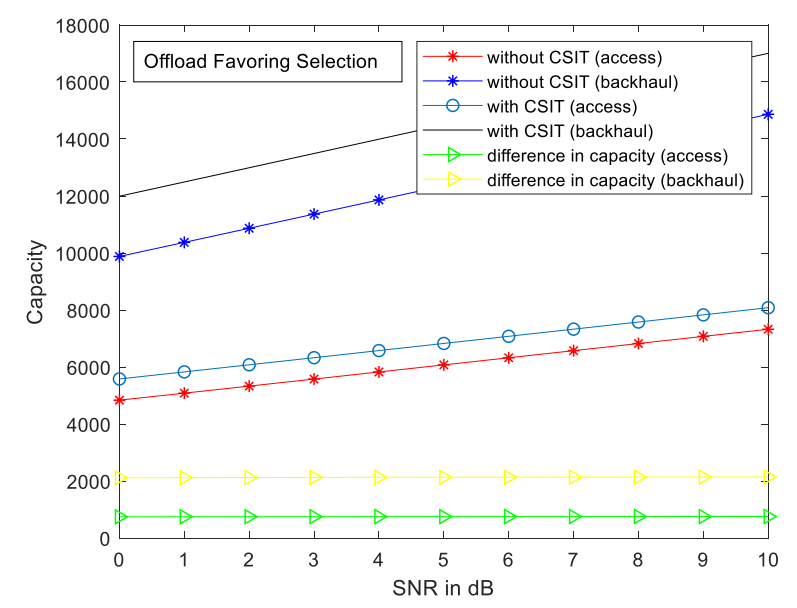

Figure 4. Effect on capacity with offload favouring random selection for access and backhaul, with and without CSIT.

Table 2. System parameters.

\begin{tabular}{|c|c|}
\hline Description & Value \\
\hline MBS transmit power & $50 \mathrm{dBm}$ \\
\hline SBS transmit power & $20 \mathrm{dBm}$ \\
\hline Power amplifier efficiency & 0.38 \\
\hline Power consumption/RF chain & $115.9 \mathrm{~mW}$ \\
\hline Idle power consumption & $45.5 \mathrm{~W}$ \\
\hline Total number of MBS antenna & 100 \\
\hline Antenna subset cases & $30,50,70$ antennas \\
\hline SINR range & $0-15 \mathrm{dBm}$ \\
\hline
\end{tabular}

\subsection{Comparison of GA Selection}

The PGA based antenna selection was compared with compared with PSO based selection [20]. Both algorithms were tested using a large data set of 30 runs with 1000 iterations per run. The population size as well the cycle limit was kept same for both the 
algorithms. The crossover percentage, mutation percentage and mutation rate was kept as $0.7,0.3$, and 0.1 respectively for PGA.

For PSO based approach an inertia weight of 1 , damping ratio of 0.99 , personal learning coefficient of 1.5 and global learning coefficient of 2 was used.

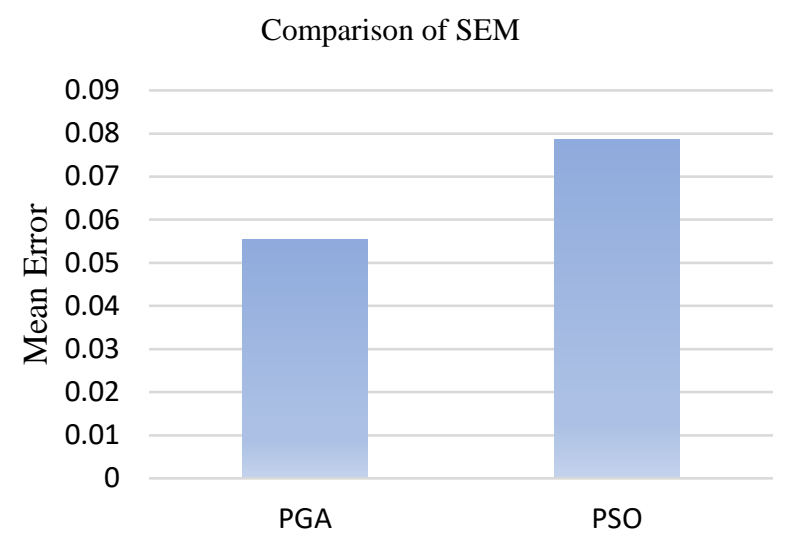

Figure 5. Comparison of SEM of PGA and PSO.

Figure 5 shows a comparison of SEM between PGA and PSO. The results clearly validate the accuracy of results.

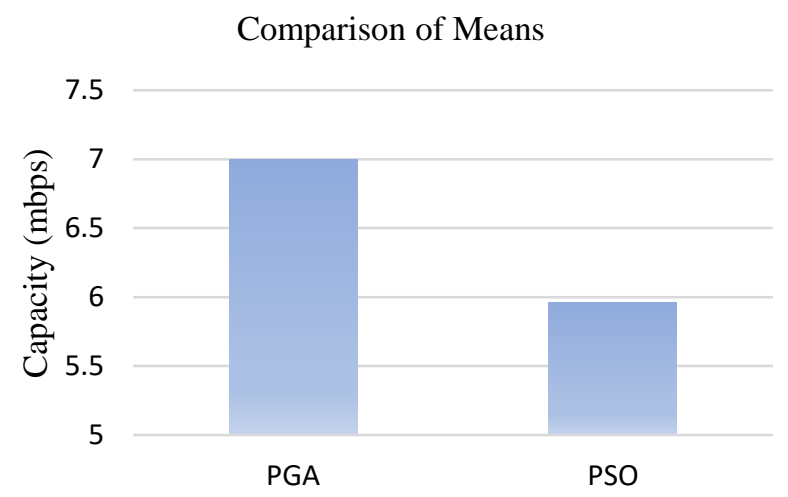

Figure 6. Comparison of mean capacity between PGA and PSO.

Figure 6 shows a comparison of mean capacity as obtained through the two algorithms, PGA and PSO. The proposed algorithm clearly out performs the PSO based antenna selection.

In Figure 7 we provide comparison of GA based antenna selection with random selection, eigenvaluebased selection and equal bandwidth partitioning. The total population size used for GA is 100 which signifies the number of MBS antennas. In random selection we choose $M_{b}>M_{a}$ where the antennas selected for access and backhaul are randomly chosen. Eigenvalue-based selection chooses antenna subset after calculating the eigenvalues of the channel matrix. A subset of the channel matrix representing maximum eigenvalues is selected which in turn represents the antenna subset being selected. These antenna selection schemes are compared with bandwidth partition where total bandwidth $W$ is divided equally for access and backhaul, i.e., $W_{a}=W_{b}=W / 2$. Since the GA based selection chooses a subset of antenna that provide the best fitness value i.e., best cost or data rate, hence, the curve for GA in Figure 7 represents the greatest capacity. Best and mean fitness value is shown in Figure 8. Cost function with respect to fitness value can be seen to have a uniform increasing trend in Figure 9.

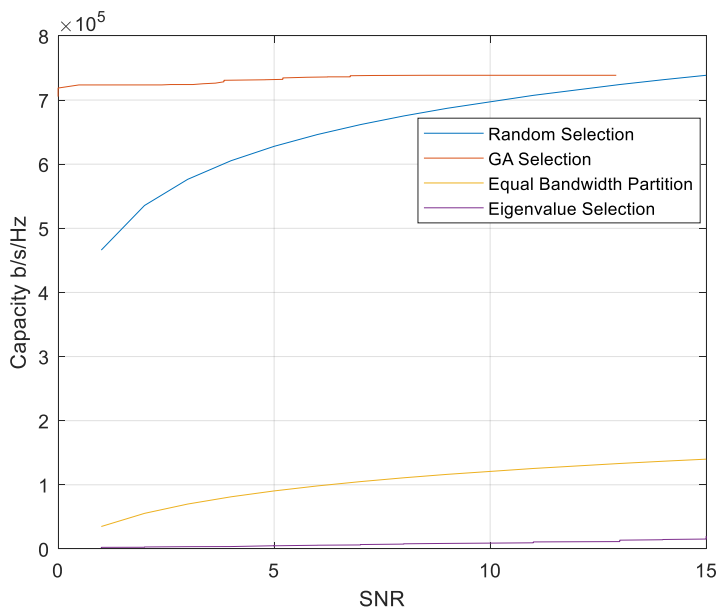

Figure 7. Comparison between different selection strategies and bandwidth selection.

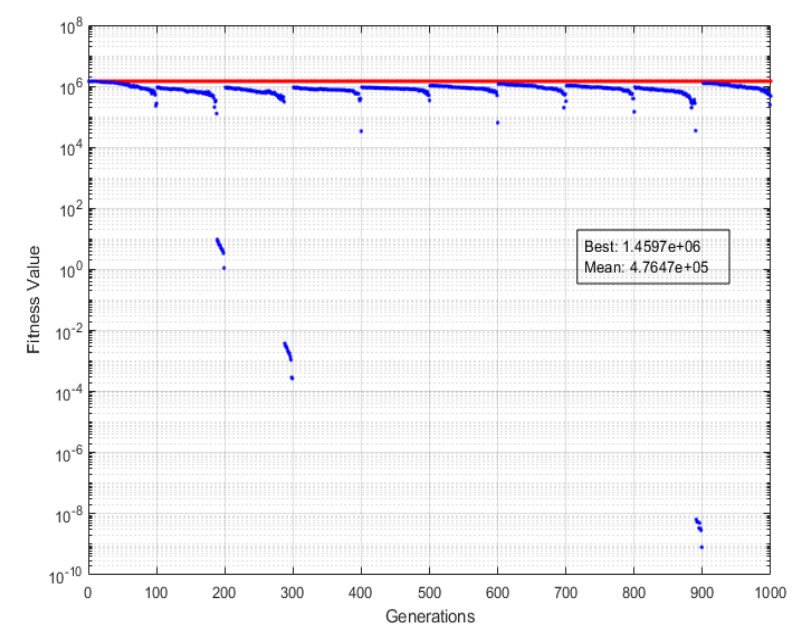

Figure 8. Fitness value vs number of generations in GA.

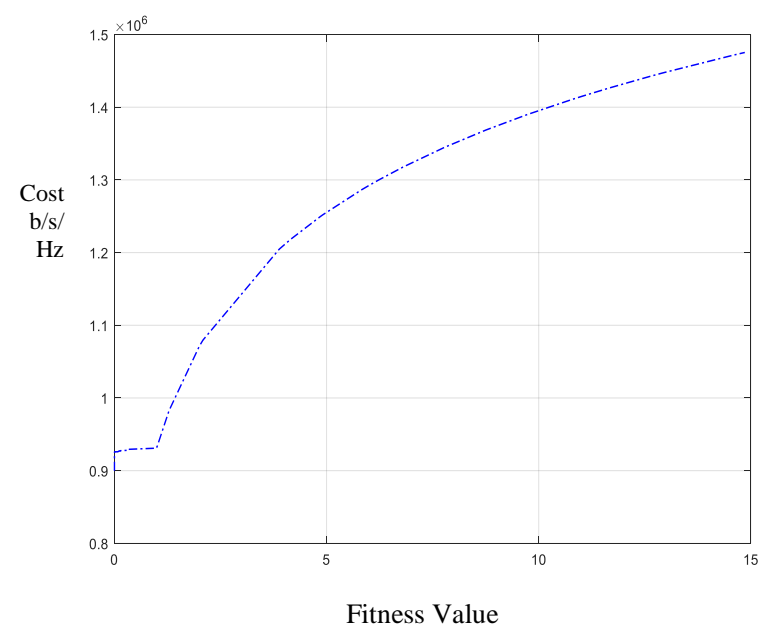

Figure 9. Fitness value vs cost in GA.

\subsection{Comparison of Antenna Partitioning and Bandwidth Partitioning}

Further, we compare bandwidth partitioning with 
antenna partitioning/selection and the results are shown in Figure 10 where the improvement in capacity with partitioning factor is much better for antenna partitioning than bandwidth partitioning. Here access partition is given as $M_{a}=(1-\eta) M$ while that for backhaul is given as $M_{b}=\eta M$, where $\eta$ is the partitioning factor. Similarly, the bandwidth partitioning as given in [34] is $W_{a}=(1-\eta) W$ and $W_{b}=\eta W$ for access and backhaul links respectively. Hence, our analysis show that antenna partitioning provides a much better capacity improvement for designing of self-backhauling 5G HetNets with massive MIMO. Similar results can be observed in Figure 11 for antenna and bandwidth partitioning for backhaul links. Hence, it can be deduced conclusively that TAS provides a more efficient IAB design strategy compared to bandwidth partitioning.

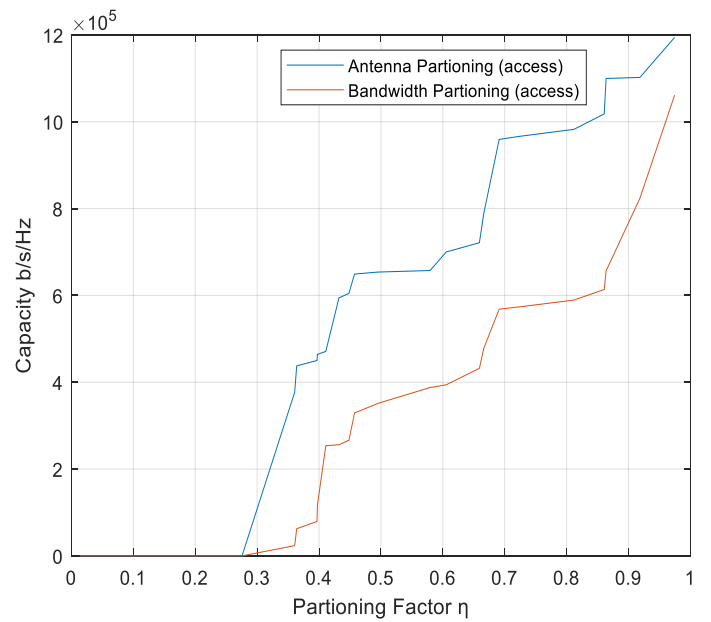

Figure 10. Antenna partitioning vs bandwidth partitioning for access link.

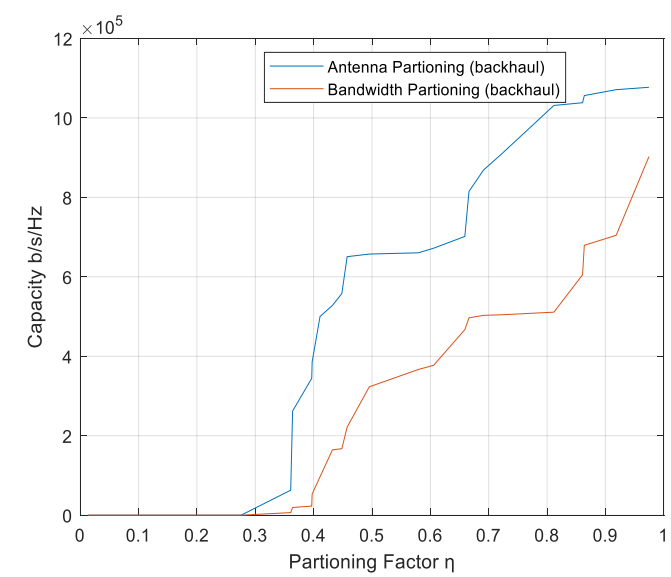

Figure 11. Antenna partitioning vs bandwidth partitioning for backhaul link.

\subsection{Mean Rate Analysis}

We consider three different cases with respect to the number of antennas selected and the number of users associated with $k^{\text {th }}$ tier. For a total of 100 MBS antennas, for case I we consider 30 transmit antennas being selected and total number of users associated with that particular tier to be 15 . In case 2 we consider 50 transmit antenna selected with 25 users served by the corresponding tier while in case 3 we consider 70 MBS transmit antennas being selected with 35 users associated with that tier. A lower number of users represents a better mean rate as the antennas being selected are the ones that provide the best possible SINR subset out of total available antennas.

It can be seen from Figure 12 that inclusion of more antennas in the subset results in lower mean rate as the demand increases in terms of number users while inclusion of less optimum subsets represents lower SINR values.

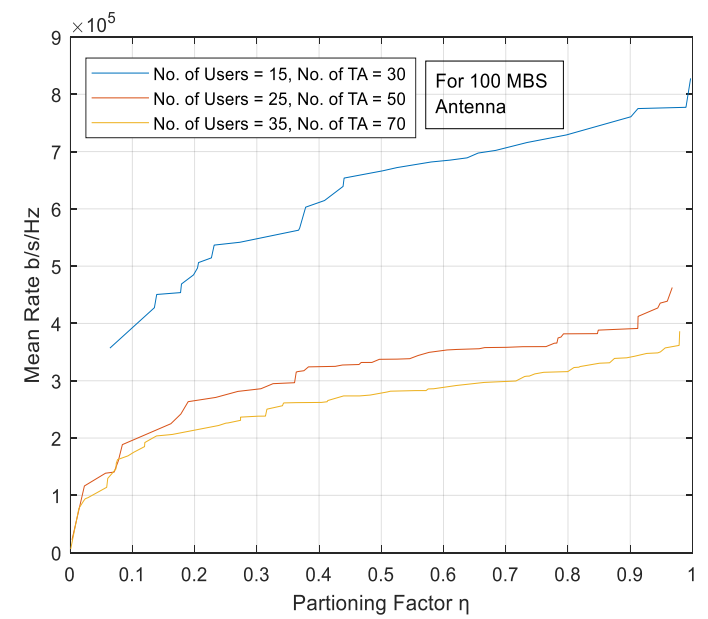

Figure 12. Mean rate analysis for three different cases of number of users and number of transmit antennas selected.

\subsection{Energy Efficiency Analysis}

Next, we analyse case 1, case 2, and case 3 for energy efficiency analysis. In Figure 13 it can be seen that energy efficiency of the network decreases for all the three cases as the number of antennas are increased beyond 30, 50, and 70 antennas respectively. This signifies the fact that power consumption of MBS increases with increase in number of transmit antennas.

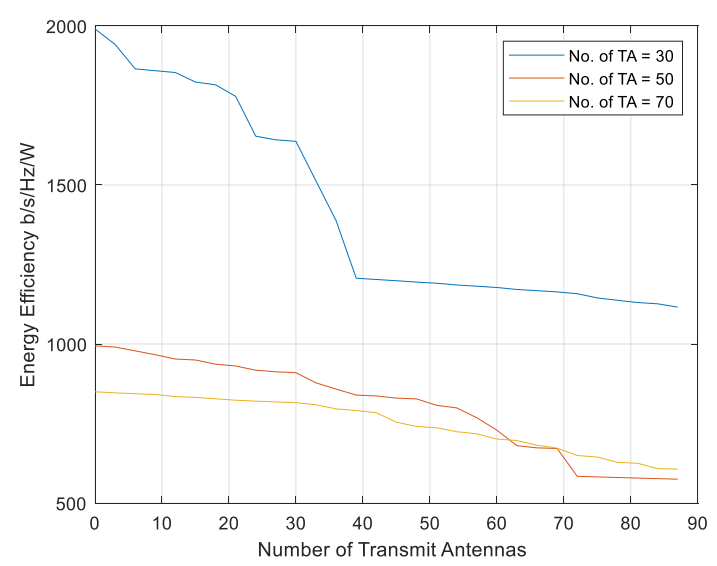

Figure 13. Energy efficiency analysis for different number of transmit antennas.

\section{Conclusions}

In this paper, we proposed a GA inspired TAS strategy 
for self-backhaul in 5G massive MIMO HetNet and investigated three different cases of selection. We developed a model for a two tier HetNet having three types of users, where the MBS is equipped with a massive number of transmit antennas. Our challenge was to investigate the effectiveness of TAS for IAB in a HetNet where TAS was GA based. We evaluated the SINR for both access and backhaul links for the proposed model and further analyzed the downlink rate coverage. A novel user association strategy was also proposed where the user association is also dependent on number of transmit antennas selection among other factors. Analytically it was shown that user association can be altered by changing the number of antennas selected per-tier. Hence, offloading MBS is possible by changing MBS antenna density per tier (in other words number of MBS antennas. The proposed work represents the following important insights:

1. The proposed GA based TAS for two tier HetNet out performs some of the conventional techniques as well as bandwidth partitioning

2. TAS based antenna selection shows promising capacity trends compared to bandwidth partitioning and hence, opens a promising research area. Our work can further be extended by incorporating instantaneous load-based analysis and comparison with other heuristic approaches for TAS along with analysis of optimum value for antenna partitioning factor.

\section{References}

[1] Agiwal M., Roy A., and Saxena N., "Next Generation 5G Wireless Networks: A Comprehensive Survey," IEEE Communications Surveys and Tutorials, vol. 18, no. 3, pp. 16171655, 2016.

[2] Aoudia I., Benharzallah S., Kahloul L., and Kazar O., "A Multi-Population Genetic Algorithm for Adaptive Qos-Aware Service Composition in Fog-Iot Healthcare Environment," The International Arab Journal of Information Technology, vol. 18, no. 3A, pp. 464-475, 2021.

[3] Bohli A. and Bouallegue R., "How to Meet Increased Capacities by Future Green 5G Networks: A Survey," IEEE Access, vol. 7, pp. 42220-422370, 2019.

[4] Cai J., Zhong R., and Li Y., "Antenna Selection for Multiple-Input Multiple-Output Systems Based on Deep Convolutional Neural Networks," PLoS ONE, vol. 14, no. 5, pp. 1-16, 2019.

[5] Celik N. and Iskander M., "Genetic-AlgorithmBased Antenna Array Design for A $60 \mathrm{~Hz}$ Hybrid Smart Antenna System," IEEE Antennas and Wireless Propagation Letters, vol. 7, pp. 795798, 2008.

[6] Chai R., Li Y., and Chen Q., "Joint Cache Partitioning, Content Placement and User
Association for D2D Enabled Heterogeneous Cellular Networks," IEEE Access, vol. 7, pp. 56642-56655, 2019.

[7] Fang W., Huang S., and Chen Y., "Genetic Algorithm-Assisted Joint Quantized Precoding and Transmit Antenna Selection in Multi-User Multi-Input Multi-Output Systems," IET Communications, vol. 5, no. 9, pp. 1220-1229, 2011.

[8] Gao Y., Vinck H., and Kaiser T., "Massive MIMO Antenna Selection: Switching Architectures, Capacity Bounds, and optimal Antenna Selection Algorithms," IEEE Transactions on Signal Processing, vol. 66, no. 5, pp. 1346-1360 2018.

[9] Ge X., Li X., Jin H., Cheng J., and Leung V., "Joint User Association and User Scheduling for Load Balancing in Heterogeneous Networks," IEEE Transactions on Wireless Communications, vol. 17, no. 5, pp. 3211-3225, 2018.

[10] Gerasimenko M., Moltchanov D., Florea R., Andreev S., Koucheryavy Y., Himayat N., Yeh S., and Talwar S., "Cooperative Radio Resource Management in Heterogeneous Cloud Radio Access Networks," IEEE Access, vol. 3, pp. 397-406, 2015.

[11] Han T., Li S., Zhong Y., Bai Z., and Kwak K., "5G Software Defined Heterogeneous Networks with Cooperation and Partial Connectivity," IEEE Access, vol. 7, pp. 72577-72590, 2019.

[12] Hanif M., Yang H., Boudreau G., Sich E., and Seyedmehdi H., "Antenna Subset Selection for Massive MIMO Systems: A Trace-Based Sequential Approach for Sum Rate Maximization," Journal of Communications and Networks, vol. 20, no. 2, pp. 144-155, 2018.

[13] Hojjati S., Ebrahimzadeh A., Najimi M., and Reihanian A., "Spectrum Selection for Cooperative Sensor Sensing in Multiantenna Sensor Networks Based on Convex Optimization and Genetic Algorithm," IEEE Sensor Journal, vol. 16, no. 10, pp. 3486-3487, 2016.

[14] Huang X., Zhang D., Tang S., Chen Q., and Zhang J., "Fairness-Based Distributed Resource Allocation in Two Tiers Heterogeneous Networks," IEEE Access, vol. 7, pp. 4000040012, 2019.

[15] Lain J., "Joint Transmit Antenna Selection for MIMO Systems: A Real-Valued Genetic Approach," IEEE Communications Letters, vol. 15, no. 1, pp. 58-60, 2011.

[16] Li C., Zhang J., Andrews J., and Letaief K., "Success Probability and Area Spectral Efficiency in Multiuser MIMO Hetnets," IEEE Transactions on Communications, vol. 64, no. 4, pp. 1544-1556, 2016. 
[17] Lu H. and Fang W., "Joint Receive Antenna Selection and Symbol Detection for MIMO Systems: A Heterogeneous Genetic Approach," IEEE Communications Letters, vol. 13, no. 2, pp. 97-99, 2009.

[18] Makki B., Ide A., Svensson T., Eriksson T., and Alouini M., "A Genetic Algorithm-Based Antenna Selection Approach for Large-But-Finite MIMO Networks," IEEE Transactions on Vehicular Technology, vol. 66, no. 7, pp. 6596595, 2017.

[19] Marinello J., Abrão T., Amiri A., Carvalho E., and Popovski P., "Antenna Selection for Improving Energy Efficiency in XL-MIMO Systems," IEEE Transactions on Vehicular Technology, vol. 69, no. 11, pp. 13305-13318, 2020.

[20] Nguyen T., Ajib W., and Assi C., "Designing Wireless Backhaul Heterogeneous Networks with Small Cell Buffering," IEEE Transactions on Communications, vol. 66, no. 10, pp. 4596-4620, 2018.

[21] Papazafeiropoulos A., Kourtessis P., Renzo M., Senior J., and Chatzinotas S., "SDN Enabled MIMO Heterogeneous Cooperative Networks with Flexible Cell Association," IEEE Transactions on Wireless Communications, vol. 18, no. 4, pp. 2037-2050, 2019.

[22] Peng X., Li J., and Zhang H., "Energy Efficient Sbss Sleep Mode Analysis for Successive User Association and Resource Scheduling Algorithms in Two Tier Heterogeneous Networks," IEEE Transactions on Wireless Communications, vol. 13, no. 9, pp. 1311-1318, 2019.

[23] Saha C. and Dhillon H., "Load Balancing in 5G HetNets with Millimeter Wave Integrated Access and Backhaul," Cornell University ArXivJourn, 2019.

[24] Saha C., Afshang M., and Dhillon H., "Bandwidth Partitioning and Downlink Analysis in Millimeter Wave Integrated Access and Backhaul for 5G," IEEE Transactions on Wireless Communications, vol. 17, no. 12, pp. 8195-8210, 2018.

[25] Sastry K., Goldberg D., and Kendall G., "Genetic Algorithms," Mathematics and Statistics Book Chapter 4, Springer, 2005.

[26] Sato Y., Campelo F., and Igarashi H., "Meander Line Antenna Design Using an Adaptive Genetic Algorithm," IEEE Transactions on Magnetics, vol. 49, no. 5, pp. 1889-1892, 2013.

[27] Sharma A., Ganti R., and Milleth J., "Joint Backhaul-Access Analysis for Full Duplex SelfBackhauling Heterogeneous Networks," IEEE Transactions on Wireless Communications, vol. 16, no. 3, pp. 1727-1740, 2017.

[28] Song X., Dong L., Wang J., Qin L., and Han X., "Energy Efficient Power Allocation for Downlink NOMA Heterogeneous Networks with Imperfect
CSI," IEEE Access, vol. 7, pp. 39329-39340, 2019.

[29] Tang H. and Nie Z., "RMV Antenna Selection Algorithm for Massive MIMO," IEEE Signal Processing Letters, vol. 25, no. 2, pp. 239-242, 2018.

[30] Wang D., Wang Y., Dong S., Huang G., Liu J., and Gao W., "Exploiting Dual Connectivity for Handover Management in Heterogeneous Aeronautical Networks," IEEE Access, vol. 7, pp. 62938-62949, 2019.

[31] Wang X., Jia M., Guo Q., Ho I., and Wu J., "Joint Power Original Bandwidth, and Detected Hole Bandwidth Allocation for Multi-Homing Heterogeneous Networks Based on Cognitive Radio," IEEE Transactions on Vehicular Technology., vol. 68, no. 3, pp. 2777-2790, 2019.

[32] Xia W., Zhang J., Jin S., Wen C., Gao F., and Zhu H., "Large System Analysis of Resource Allocation in Heterogeneous Networks with Wireless Backhaul," IEEE Transactions on Communications, vol. 65, no. 11, pp. 50405053, 2017.

[33] Xu W., Qiu R., and Jiang X., "Resource Allocation in Heterogeneous Cognitive Radio Network with Non-Orthogonal Multiple Access," IEEE Access, vol. 7, pp. 57488-57499, 2019.

[34] Zhong S., Feng H., Zhang P., Xu J., Luo H., Zhang J., Yuan T., and Huang L., "Deep Learning Based Antenna Selection for MIMO SDR Systems," Sensors, vol. 20, no. 23, 2020.

[35] Zhou J., Zhang X., and Wang W., "Joint Resource Allocation and User Association for Heterogeneous Service in Multi-Access Edge Computing Networks," IEEE Access, vol. 7, pp. 12272-12282, 2019. 


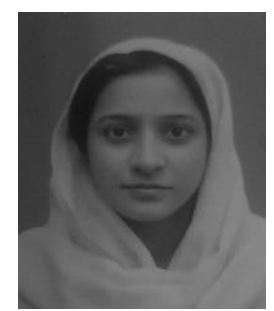

Farah Akif received B. Sc. Electrical Engineering from UET Peshawar in 2006, M.Sc from UET Peshawar in 2010 and is currently pursuing $\mathrm{Ph}$. D. from Islamic International University, Islamabad with major in communication. She has many IEEE International conference publications along with some International journal publications. She has over nine years of teaching experience at UET. Her major areas of interest include wireless communication, heterogeneous networks, wireless sensor networks and computer networks.

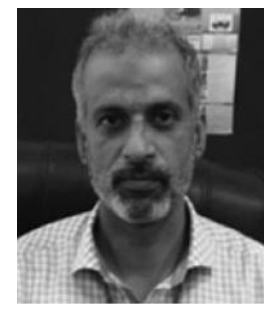

Aqdas Malik received Ph.D. degree from MAJU, Pakistan, in 2006. He has over 15 years post $\mathrm{Ph}$. D. experience in teaching and research at different institutions of good repute in Pakistan. More than three $\mathrm{Ph}$. Ds have been produced under his supervision. $\mathrm{He}$ is currently the Vice President of Higher Studies \& Research (HS\&R). His research interests include digital/wireless communications, digital signal processing, information, and power electronics.

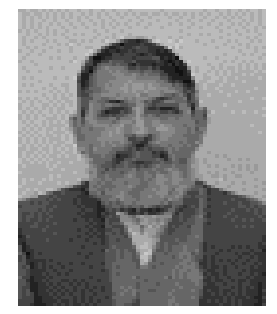

Ijaz Qureshi received B. E. degree in avionic engineering from NED University Karachi, Pakistan, the M.S. degree from Department of Electrical Engineering METU, Ankara, Turkey in 1980. He did his Ph.D. from University of Toronto, Canada, in 1985. He has worked as professor at various universities of Pakistan including Quaid -e- Azam University (1987-2000), Muhammad Ali Jinnah University (2002-2007), International Islamic University (IIU) (2007-2009). Since 2009, he has been working as a professor and in charge graduate program with Department of Electrical Engineering, Air University, Islamabad, Pakistan. He is also the director of Institute of Signals, systems and software computing (ISSS), Islamabad, Pakistan. $\mathrm{He}$ has around 300 publications in various fields of engineering. His research interests are digital communication, radar signal processing, image processing, soft computing and engineering computational mathematics. Most unfortunately, Dr. Qureshi passed away in January 2021.

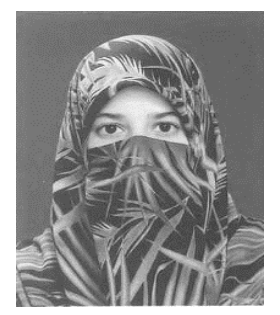

Ayesha Abbasi did her B. Sc. From Air University in 2008. She did masters in Electrical Engineering from National University of Science and Technology (NUST), Islamabad in 2013. She is a lecturer in Department of Electrical Engineering at International Islamic University (IIU), Islamabad. Her work mainly focuses on energy management systems in micro grids. She is currently enrolled in Ph. D. EE at IIU. 